\title{
Jaunissement Mortel du Cocotier et Mutations Sociales à Grand-Lahou, Côte d'Ivoire
}

\author{
Tenguel Sosthène N'guessan, PhD \\ Université Nangui Abrogoua/Unité de Formation et de Recherche des \\ Sciences et Gestion de l'Environnement, Côte d'Ivoire \\ Centre Suisse de Recherches Scientifiques, Côte d'Ivoire \\ Daniel Kra Kouame, PhD \\ Guéhi Jonas Ibo, PhD \\ Hortense Atta Diallo Taky, PhD \\ Université Nangui Abrogoua/Unité de Formation et de Recherche des \\ Sciences de la Nature, Côte d'Ivoire
}

Doi:10.19044/esj.2019.v15n25p130 URL:http://dx.doi.org/10.19044/esj.2019.v15n25p130

\section{Résumé}

La culture du coco constitue un pan important de l'économie ivoirienne et régente la vie des populations qui la cultivent. Cependant le jaunissement mortel du cocotier (JMC), intervenu dans les plantations ivoiriennes de Grand-Lahou a sapé les fondements de l'économie sociale et la dynamique sociale par leurs effets pervers. Afin de cerner les implications sociales du jaunissement mortel du cocotier, une étude a été conduite dans une dizaine de villages de Grand-Lahou sujets à cette épidémie. La collecte de données quantitatives a été effectuée auprès de 101 personnes déterminées par l'échantillonnage par quotas. Celles qualitatives ont été réalisées auprès de 10 femmes et 10 hommes tous impliqués dans la production et la transformation de la noix de coco. Pour le traitement et l'analyse des données quantitatives, il a été mobilisé le logiciel Statistical Package for Social Sciences (S.P.S.S) tandis que l'usage de MaxQDA a été fait pour les données qualitatives. Les résultats attestent d'une espérance de vie suffisante des répondants pour apprécier l'impact du JMC. Les implications du jaunissement mortel du cocotier portent sur le bouleversement du système traditionnel de désignation de l'autorité coutumière $(81,8 \%)$, l'absence de développement locale conséquence de la perte de revenus $(15,8 \%)$ et des superficies de cocoteraies $(83,2 \%)$, ainsi que le relâchement des relations intergénérationnelles aînéscadets. Malgré son faible impact sur la polygamie comme construit culturel, le JMC participe à la transformation des relations de genre favorisant l'accès de la femme à la terre et son autonomie. En définitive, le JMC se présente comme un fait social participant à l'essor de la femme mais jetant les jalons 
d'un affaiblissement de la conscience collective.

Mots-clés : Jaunissement mortel du cocotier, Mutation sociale, Grand-Lahou

\title{
Coconut Yellowing and Social Mutations In Grand-Lahou, Ivory Coast
}

\author{
Tenguel Sosthene N'guessan, PhD \\ Universite Nangui Abrogoua/Unite de Formation et de Recherche des \\ Sciences et Gestion de l'Environnement, Côte d'Ivoire \\ Centre Suisse de Recherches Scientifiques, Côte d'Ivoire
}

Daniel Kra Kouame, PhD

Guéhi Jonas Ibo, PhD

Hortense Atta Diallo Taky, PhD

Universite Nangui Abrogoua/Unite de Formation et de Recherche des

Sciences de la Nature, Côte d'Ivoire

\begin{abstract}
Coconut farming is an important part of the Ivorian economy and governs the lives of people who grow it. However, the Lethal Yellowing Disease (LYD), occurring in the Ivoirian plantations of Grand-Lahou has undermined the foundations of the social economy and the social dynamics by their perverse effects. In order to identify the social implications of the fatal yellowing of the coconut tree, a study was conducted in ten villages in GrandLahou subject to this epidemic. The collection of quantitative data was carried out among 101 people determined by quota sampling. Qualitative survey was conducted among 10 women and 10 men all involved in the production and processing of coconut. For data managment, S.P.S.S for quantitative data and MaxQDA for qualitative data were used. The results attest to the respondents' sufficient life experience to appreciate the impact of the LYD. The consequences of the Lethal Yellowing Disease relate to the disruption of the traditional customary designation system (81.8\%), the lack of local development resulting from the loss of income (15.8\%) and areas of plantation plantations $(83.2 \%)$, the relaxation of intergenerational relations between seniors and cadets. Despite its weak impact on polygamy as a cultural construct, the LYD participates in the transformation of gender relations which promote women's access to land and their autonomy. Ultimately, the JMC is presented as a social fact participating in the development of women
\end{abstract}


but laying the foundation for a weakening of the collective consciousness.

Keywords: Fatal Yellowing of the Coconut Palm, Social mutation, GrandLahou

\section{Introduction}

L'adoption de la culture du cocotier comme culture commerciale par les populations des zones côtières d'Afrique de l'Ouest et de l'Est revêt pour les ménages d'agriculteurs de cette frange littorale un intérêt triple : consommation familiale, trésorerie « sur tronc » (on vend des noix quand on a besoin d'argent) ou garantie pour les crédits (la production est gagée en échange d'un crédit octroyé pour résoudre des besoins ponctuels) et source de revenus (Bene et al., 2009 ; Dollet et al., 2009). Elle occupe, de ce fait, une place de choix dans la vie des populations en termes d'usages sociaux et économiques ; l'activité impliquant en effet la communauté entière à l'échelle du village avec un rôle prépondérant joué par les femmes (Gachanja et al., 2007).

Implantées pour la plupart sur le littoral ivoirien à l'instar des autres pays de l'Afrique de l'Ouest comme le Ghana et le Togo par la SODEPALM chargée du Plan Cocotier en 1966 (Amagou \& Brunin, 1974), les cocoteraies occupent 46000 hectares de terres cultivables en Côte d'Ivoire (Amrizal, 2003). Principale culture de rente pour plus douze mille cinq cents familles (Ouvrier et al., 1995) et principale source de revenus pour plus de vingt mille familles de la basse Côte d'Ivoire (Assa et al., 2006), le cocotier a ainsi participé à l'embellie économique du pays et à une stabilité financière des ménages, transformant ainsi leur quotidien.

Déclarée à Grand-Lahou en Côte d'Ivoire en 2013, mais dont les origines remonteraient en 1997 (Yao et al., 2012) dans les plantations de la Société Ivoirienne de Coco Râpé (SICOR), la maladie du jaunissement mortel du cocotier (JMC) ou Lethal Yellowing Disease, dû à des phytoplasmes, est un important facteur limitant de la filière du cocotier (Legoupil \& Courbet, 2008). Surnommée « maladie mystérieuse » ou encore « sida du cocotier » par les populations ivoiriennes, le JMC a détruit de nombreuses cocoteraies au cours des dernières décennies (Mariau, 1999) dont 350 hectares du vaste verger ivoirien dans le département de Grand-Lahou. Cette maladie a entraîné une perte de la production de noix de coco et de coprah estimée à plus de deux milliards de francs CFA en Côte d'Ivoire (Nkaka, 2013), et laissé des populations sans ressources, sapant ainsi par leurs effets pervers la dynamique sociale.

Si les effets du JMC restent prolixes aux plans agronomique et économique, la littérature est peu fournie, à l'exception de quelques écrits (Ruf et al., 2009), quant aux retombées sociales notamment sur la dynamique 
sociétale. Comment le JMC a-t-il joué sur la dynamique sociale et les relations de genre ? Tel est le propos de cet article qui analyse les effets pervers du JMC sur l'organisation sociale des populations de Grand-Lahou.

\section{Méthodologie}

L'étude est basée sur une enquête de terrain réalisée dans les localités de Badadon, Braffedon, Doudougbazou, Gredjiberi, Lahou-Kpanda, Liboli, Likpilasse, Village 1, Village 2 et Yaokro du département ayant subi les affres du jaunissement mortel du cocotier en Juin 2016.

La technique de collecte de données a combiné les approches quantitative et qualitative (Robert \& Bouillarguet, 1997 ; N'da, 2002 ; Schumacher, 2002). L'approche quantitative a été conduite auprès de 101 personnes déterminées à l'aide d'un échantillonnage par quotas. L'approche qualitative a été réalisée auprès de 20 personnes dont 10 femmes et 10 hommes tous impliqués dans la production et la transformation de la noix de coco.

Pour la base des données quantitatives des observations répertoriées, recours a été fait au tableur Microsoft Excel ; et les analyses statistiques, les organisations et les présentations de tableaux et graphiques ont été effectuées grâce au logiciel d'analyse Statistique S.P.S.S v21. Quant aux données qualitatives, une analyse de contenu à l'aide de MaxQDA a permis de mettre en exergue une grille thématique. Celle-ci a permis, à l'aide de l'analyse structurelle, d'aboutir à une configuration signifiante des thèmes les plus fréquemment évoqués par les enquêtés.

\section{Cadre de l'étude}

Située à environ 150 kilomètres d'Abidjan capitale économique de la Côte d'Ivoire, Grand-Lahou, zone d'étude, est arrosée par le fleuve Bandadama, la lagune et l'Océan atlantique. Elle est située au Sud du pays, au bord du golfe de Guinée, à l'embouchure du fleuve Bandama. Le département de Grand-Lahou a une population estimée à 151313 habitants. La ville compte environ 67483 habitants dont 44,59\% de femmes (INS, 2014). Les autochtones, les Avicam appelés aussi Brignan font partie du groupe ethnoculturel des Akan (Gaston, 1910). Ils sont appelés, avec les Abouré, les Ebrié, les Adjoukrou, les Ahizy et les Alladjan, les Akan lagunaires. L'économie de Grand-Lahou est dominée par la pêche, l'agriculture et le commerce. La culture dominante dans ce groupe ethnoculturel est le cocotier qui a permis la mise en place d'une infrastructure économique permettant de 
consolider l'action sociale et la prise en charge d'un développement durable de ses populations, mais infecté par la maladie du jaunissement mortel.

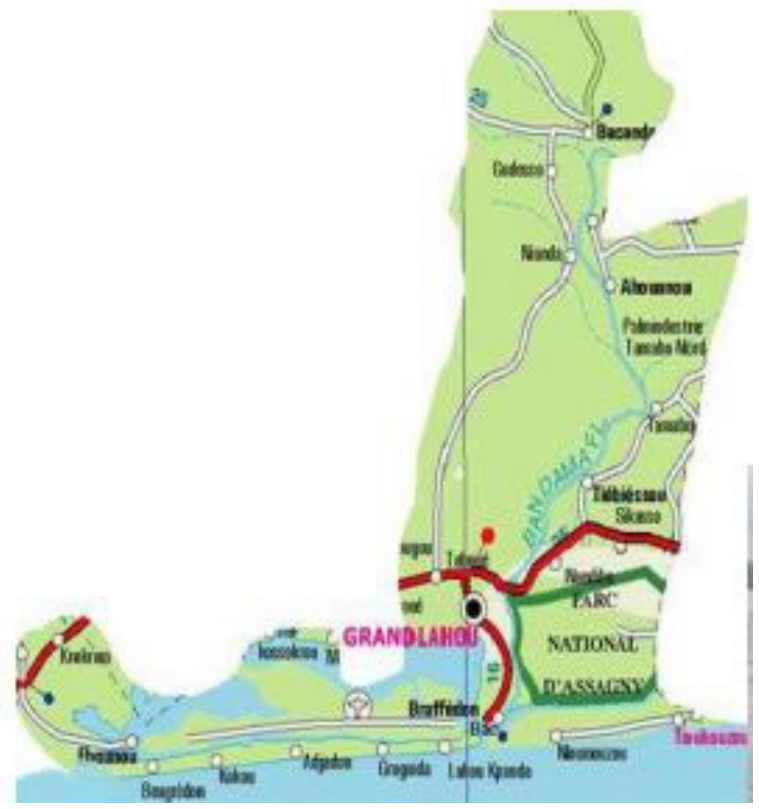

Carte 1: Zone d'étude

\section{Résultats}

\section{Caractéristiques sociodémographiques des enquêtés}

L'enquête auprès des ménages a concerné 101 personnes dont 85,1\% d'hommes et $14,9 \%$ de femmes (Tableau 1). L'importance des hommes tient principalement aux construits socioculturels autour du travail de la terre, axés sur la virilité des travaux champêtres et le fait pour l'homme de satisfaire aux besoins sociaux de la famille. Cet intérêt pour la terre explique l'importance relative de la population agricole représentant $64,4 \%$ des enquêtés et s'inscrit dans la vision de la politique économique dont l'agriculture constitue le principal pilier. Du fait de la politique agricole qui a accordé une place de choix à la main-d'œuvre extérieure, une forte présence des populations allogènes dans les localités de l'enquête qui représentent 30,7\% de la population enquêtée a été observée. Seulement un dixième de la population agricole est de sexe féminin (10,8\%). L'âge moyen des personnes enquêtées est de 56,3 ans avec une durée de présence moyenne, dans les lieux d'enquêtes, de 20 ans pour $72,3 \%$ des répondants. Ces deux résultats ont montré que les enquêtés ont une espérance de vie suffisante pour apprécier dans la durée les mutations en cours dans leur société. Près de $90,0 \%$ des hommes enquêtés sont mariés alors que cette proportion est de 10,2\% chez les femmes. Le niveau d'instruction reste faible dans la mesure où plus de la moitié des répondants est non scolarisée $(53,5 \%)$. 
Tableau 1: Caractéristiques sociodémographiques des répondants

\begin{tabular}{|c|c|c|c|c|c|c|c|}
\hline \multirow{2}{*}{\multicolumn{2}{|c|}{$\begin{array}{c}\text { Caractéristiques } \\
\text { sociodémographiques }\end{array}$}} & \multicolumn{2}{|c|}{ Féminin } & \multicolumn{2}{|c|}{ Masculin } & \multicolumn{2}{|c|}{ Total } \\
\hline & & Effectif & Pourcentage & Effectif & Pourcentage & Effectif & Pourcentage \\
\hline \multirow{6}{*}{ Age } & $20-29$ ans & 0 & $0,0 \%$ & 1 & $100,0 \%$ & 1 & $1,0 \%$ \\
\hline & $30-39$ ans & 1 & $8,3 \%$ & 11 & $91,7 \%$ & 12 & $11,9 \%$ \\
\hline & $40-49$ ans & 4 & $21,1 \%$ & 15 & $78,9 \%$ & 19 & $18,8 \%$ \\
\hline & $50-59$ ans & 3 & $11,5 \%$ & 23 & $88,5 \%$ & 26 & $25,7 \%$ \\
\hline & $>60$ ans & 7 & $16,3 \%$ & 36 & $83,7 \%$ & 43 & $42,6 \%$ \\
\hline & Total & 15 & $14,9 \%$ & 86 & $85,1 \%$ & 101 & $100,0 \%$ \\
\hline \multicolumn{2}{|l|}{ Age moyen } & \multicolumn{2}{|c|}{55,4 ans } & \multicolumn{2}{|c|}{56,5 ans } & \multicolumn{2}{|c|}{56,3 ans } \\
\hline \multirow{6}{*}{$\begin{array}{c}\text { Situation } \\
\text { matrimoniale }\end{array}$} & Célibataire & 2 & $25,0 \%$ & $\bar{~} 6$ & $75,0 \%$ & $\overline{88}$ & $\mathbf{7 , 9 \%}$ \\
\hline & Concubin(e) & 3 & $12,5 \%$ & 21 & $87,5 \%$ & 24 & $23,8 \%$ \\
\hline & Marié(e) & 6 & $10,2 \%$ & 53 & $89,8 \%$ & 59 & $58,4 \%$ \\
\hline & Veuf/Veuve & 3 & $33,3 \%$ & 6 & $66,7 \%$ & 9 & $\mathbf{8 , 9 \%}$ \\
\hline & Séparé & 1 & $100,0 \%$ & 0 & $0,0 \%$ & 1 & $1,0 \%$ \\
\hline & Total & 15 & $14,9 \%$ & 86 & $85,1 \%$ & 101 & $100,0 \%$ \\
\hline \multirow{5}{*}{$\begin{array}{l}\text { Niveau } \\
\text { d'instruction }\end{array}$} & Aucun & 7 & $13,0 \%$ & 47 & $87,0 \%$ & 54 & $53,5 \%$ \\
\hline & Primaire & 4 & $19,0 \%$ & 17 & $81,0 \%$ & 21 & $20,8 \%$ \\
\hline & Secondaire & 3 & $13,0 \%$ & 20 & $87,0 \%$ & 23 & $22,8 \%$ \\
\hline & Supérieur & 1 & $33,3 \%$ & 2 & $66,7 \%$ & 3 & $3,0 \%$ \\
\hline & Total & 15 & $14,9 \%$ & 86 & $85,1 \%$ & 101 & $100,0 \%$ \\
\hline \multirow{3}{*}{ Nationalité } & Autochtone & 13 & $18,6 \%$ & 57 & $81,4 \%$ & 70 & $69,3 \%$ \\
\hline & Allogène & 2 & $6,5 \%$ & 29 & $93,5 \%$ & 31 & $30,7 \%$ \\
\hline & Total & 15 & $14,9 \%$ & 86 & $85,1 \%$ & 101 & $100,0 \%$ \\
\hline \multirow{9}{*}{$\begin{array}{l}\text { Statut socio- } \\
\text { professionnel }\end{array}$} & Sans activité & 1 & $20,0 \%$ & 4 & $80,0 \%$ & 5 & $5,0 \%$ \\
\hline & $\begin{array}{l}\text { Leader } \\
\text { communautaire }\end{array}$ & 2 & $20,0 \%$ & 8 & $80,0 \%$ & 10 & $9,9 \%$ \\
\hline & Commerçant & 1 & $14,3 \%$ & 6 & $85,7 \%$ & 7 & $6,9 \%$ \\
\hline & Cultivateur & 7 & $10,8 \%$ & 58 & $89,2 \%$ & 65 & $64,4 \%$ \\
\hline & Employé & 0 & $0,0 \%$ & 2 & $100,0 \%$ & 2 & $2,0 \%$ \\
\hline & Enseignant & 1 & $33,3 \%$ & 2 & $66,7 \%$ & 3 & $3,0 \%$ \\
\hline & Ménagère & 3 & $100,0 \%$ & 0 & $0,0 \%$ & 3 & $3,0 \%$ \\
\hline & Pêcheur & 0 & $0,0 \%$ & 6 & $100,0 \%$ & 6 & $5,9 \%$ \\
\hline & Total & 15 & $14,9 \%$ & 86 & $85,1 \%$ & 101 & $100,0 \%$ \\
\hline
\end{tabular}

\section{Niveau de connaissance du jaunissement mortel du cocotier}

Dans un environnement économique marqué par la culture du cocotier, les effets pervers associés à la pratique culturale ne sauraient passer inaperçus chez les populations. Il ressort de l'analyse du graphique 1, que les populations enquêtées ont un niveau de connaissance suffisant de la maladie du jaunissement mortel du cocotier (JMC). En effet, près de neuf répondants sur dix $(85,1 \%)$ ont déjà entendu parler de la maladie et $63,4 \%$ ont déclaré la connaître. Ces résultats traduisent dans les faits l'étroitesse des liens entre les répondants et la culture du cocotier d'une part, et d'autre part leur expérience de la cocoteraie. 


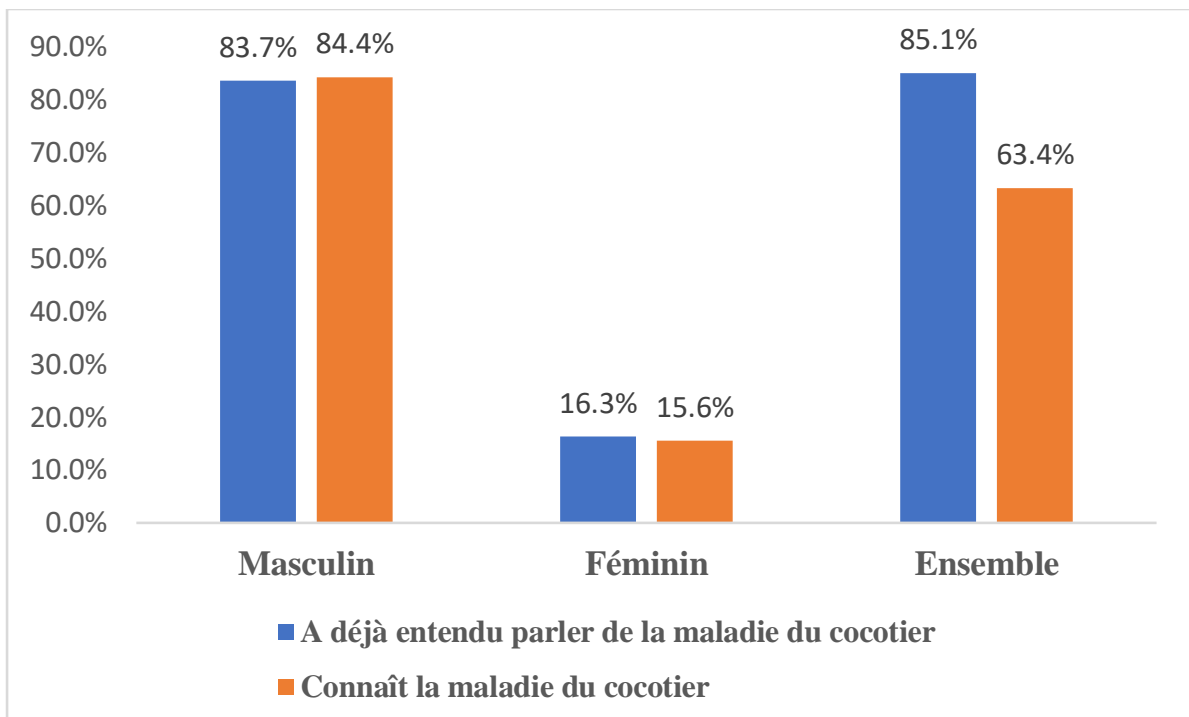

Graphique 1: Niveau de connaissance des répondants du jaunissement mortel du cocotier

Implication du jaunissement mortel du cocotier dans la vie de la communauté La gouvernance traditionnelle du peuple Avicam repose sur le principe gérontocratique. Ce sont les aînés des lignages qui conduisent les affaires de la communauté. À l'image des autres lagunaires de Côte d'Ivoire, la gouvernance des Avikam est constituée de démocraties villageoises, c'està-dire que leur unité politique et sociale la plus importante est le village. Traditionnellement, jusqu'au XVIIIème siècle, le peuple Avikam était placé sous l'autorité d'un chef qui résidait à Afouavo et disposait d'un pouvoir s'étendant aux domaines militaire, politique, spirituel et judiciaire. Au milieu du XIXème siècle, il disparaît et toute autorité individuelle avec lui. De nouvelles structures apparaissent : l'èra, l'ègbata, l'èdon et l'èsigban. L'èra, c'est-à-dire le village, est placé sous l'autorité d'un èdonsa ou chef du village, doublé d'un conseil représentatif. L'existence de plusieurs clans dans le village implique une direction collective. Le Conseil, composé des représentants des clans et des lignages constitutifs du village, assiste l'èdonsa dans la gestion des affaires publiques.

Selon les répondants ayant participé à l'enquête, la maladie du JMC n'aurait pas occasionné un réel dysfonctionnement dans la gouvernance locale. Pour autant, parmi les 9,2\% attestant du contraire (Graphique 2), le JMC est à l'origine d'une désorganisation du fonctionnement du village. L'autorité coutumière est mise à mal par des coups de force au sein de la notabilité. Attestée par $81,8 \%$ de ces répondants, la maladie du JMC a bouleversé le système traditionnel de désignation de l'autorité coutumière reposant traditionnellement sur un consensus exprimé par $33,7 \%$ des répondants. Les coups de force au sein de la notabilité sont la conséquence de 
la perception de la passivité de l'autorité traditionnelle face à une maladie compromettant le bien-être économique et social des populations.

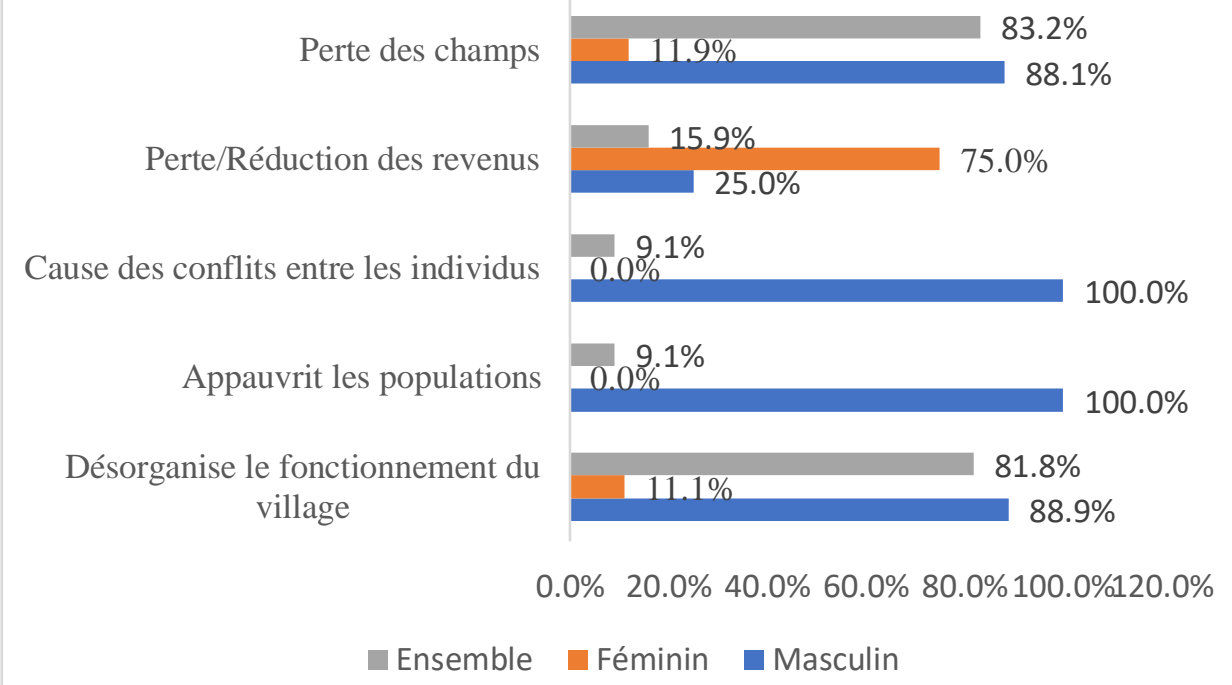

Graphique 2: Impact du jaunissement mortel du cocotier dans le quotidien des villages producteurs

L'analyse met par ailleurs en évidence l'appauvrissement à grande échelle des planteurs de coco du fait du jaunissement mortel du cocotier. L'activité économique reposant essentiellement sur la culture du coco, un ralentissement de cette activité est perçu par $9,0 \%$ des répondants. Il en résulte un arrêt du développement des villages exprimé par 31,0\% des répondants. En sus, six individus sur dix attestent de l'appauvrissement général de la population. Le sentiment que «plus rien ne va dans le village » est perçu par neuf répondants sur dix depuis l'avènement du jaunissement mortel du coco. L'envers de cette perception se traduit sur le plan économique par la perte de revenus $(15,8 \%)$ et des surfaces de cocoteraies $(83,2 \%)$.

Au plan social, les conséquences sont faiblement mises en exergue par les populations. Le jaunissement mortel du cocotier a favorisé un exode rural à moindre échelle. Les déplacements des populations principalement effectués par la jeunesse vers des zones perçues comme offrant plus d'opportunités d'épanouissement social contribuent au ralentissement de la vie sociale et à l'exacerbation de l'enclavement des villages. Par ailleurs une modification de la gestion foncière exprimée par 8,9\% des répondants est observée. De plus, seulement $4,5 \%$ des répondants témoignent de conséquences sur le statut matrimonial.

Reconstruction des rapports de genre face au jaunissement mortel du cocotier 
Les relations de genre sont en général des construits sociaux; lesquels construits ont pour réceptacle les pratiques culturelles qui se présentent en général comme des faits sociaux. Chez les peuples Avicam et les populations enquêtées, la polygamie fait partie des construits sociaux qui régentent le cadre matrimonial. Sous cet angle, le jaunissement mortel du cocotier n'a nullement altéré ce fait social chez les planteurs de cette culture. Malgré les effets économiques désastreux du JMC, 66,3\% des planteurs sont demeurés polygames. Il est par ailleurs observé une stabilité des couples existants. Seulement $9,9 \%$ des couples ont connu un divorce du fait du manque d'argent $(50,0 \%)$ et de la pauvreté $(33,3 \%)$ induits par le JMC.

En ce qui concerne les relations de genre ou entre homme et femme, environ le tiers des répondants ont prétendu que le JMC a entaché lesdites relations. Les hommes $(89,3 \%)$ plus que les femmes $(10,7 \%)$ ont confirmé ces changements. Pour les hommes, ces changements se traduisent par le nonrespect à eux accordé par leurs conjointes du fait de l'amenuisement de leurs ressources financières. Cette réduction du pouvoir financier participe de l'effritement de leur autorité conjugale et rend les relations difficiles voire conflictuelles avec leurs conjointes. Du point de vue des femmes, les changements majeurs induits par le JMC portent sur leur autonomie manifestée par la pratique d'activités génératrices de revenus.

Implication foncière du jaunissement mortel du cocotier

La terre en milieu rural ivoirien constitue la principale source pourvoyeuse de revenus pour les populations. L'accès à la terre est de ce fait régi par des règles sociales qui structurent les relations de genre et déterminent le niveau de propriété au sein des familles.

Les Avikam ont pour principales sources de richesse la terre et l'eau. Tous les sols sont mis en valeur. Les familles les exploitent sans avoir la propriété. Les terres deviennent des biens du lignage selon diverses procédures : droit des premiers occupants, annexion par alliance matrimoniale ou occupation par la force. La cour ou èva est le siège de l'activité économique. L'èva, unité de production et de consommation, est un segment de lignage concentré en un même lieu. Les activités de production : culture, pêche, chasse, s'organisent sous l'autorité du chef de cour ou èva.

Autant pour les hommes que pour les femmes, les possibilités d'accès à la terre concernent la requête formulée à l'endroit du chef de famille $(41,0 \%)$, l'héritage $(21,0 \%)$, la location $(16,0 \%)$ et dans une moindre mesure par l'achat $(2,0 \%)$. Selon les répondants en effet, le JMC n'a nullement entamé la logique sociale d'accès à la terre. Toutefois le JMC a induit des effets positifs au profit des femmes. Le droit de propriété coutumière de la terre est ainsi accordé aux femmes selon 57,4\% des répondants. En sus, huit répondants sur dix attestent de la liberté accordée à la femme de créer ses propres plantations avec en prime le choix de la culture à produire $(78,2 \%)$. Ces résultats mettent en 
exergue une inversion de la tendance en termes de restauration des droits des femmes, de l'égalité entre hommes et femmes et de la revalorisation de la position sociale de la femme.

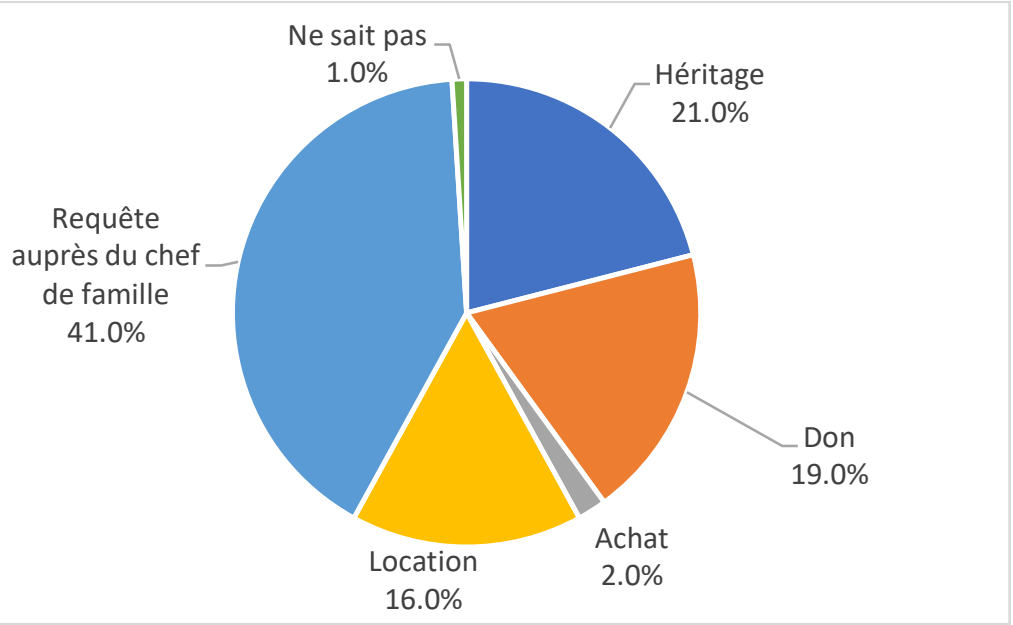

Graphique 3: Modalités d'accès à la terre selon les répondants

\section{Dynamiques sociales intergénérationnelles aînés/cadets}

Dans la culture Avicam, la dynamique sociale intergénérationnelle entre aînés et cadets repose sur un principe fondamental : le respect de l'aîné. Cette obéissance aux anciens, structure les rapports sociaux. Il ressort de la vie communautaire en effet l'emprise des aînés sur les jeunes : le changement de statut matrimonial (passage du statut d'enfant à l'adulte ou de célibataire à marié) tout comme la couverture de l'ensemble des besoins sociaux du jeune relève de la responsabilité de l'ancien, détenteur quasi exclusif de la richesse familiale, qui de ce fait lui donne pouvoir et autorité sur le jeune.

Avec l'avènement du JMC, ce principe tendrait à s'affaiblir. L'inversion de la dynamique économique du fait du JMC postule implicitement l'affectation de la dynamique sociale entre aîné/cadet. Les résultats de l'étude ont montré en effet que les rapports intergénérationnels ont connu une forme de relâchement. Le changement de comportement envers les aînés est exprimé par près de $40 \%$ des enquêtés. Ces changements de comportement s'analysent en termes d'insoumission et de désobéissance que confirment $13,9 \%$ des répondants. Celles-ci s'enracinent dans la perte du pouvoir monétaire qui a longtemps structuré les rapports sociaux du fait de l'embellie financière occasionné par la productivité des cocoteraies. Face au relâchement du pouvoir économique, certaines valeurs sociales ayant cours tendent parallèlement à perdre leur poids dans la communauté : le respect des aînés fait ainsi place au non-respect, l'arrogance cultivée par les jeunes s'apparente à une forme de révolte face aux incapacités manifestées par les aînés. Le droit d'aînesse ou l'autorité mise à mal par le JMC se prolonge dans 
l'incapacité des aînés à retenir la plupart des jeunes dans les villages. L'exode rural des jeunes a été attesté par près de la moitié $(45,5 \%)$ des enquêtés. Par ailleurs, les effets pervers du JMC se traduisent par la transgression de certaines normes sociales avec l'adultère dont $15,8 \%$ des jeunes en sont auteurs.

\section{Conclusion}

La présente étude a analysé les implications du jaunissement mortel du cocotier sur la dynamique sociale. L'étude a renseigné qu'au-delà des conséquences économiques liées au JMC, traduites par la réduction du pouvoir d'achat des populations, les implications sociales semblent indéniables et concourent à la transformation des relations de genre chez les Avicam. Malgré des attitudes subversives tendant à destituer l'autorité traditionnelle, conséquence d'une perception de sa passivité face au JMC, la gouvernance sociétale reste dominée par les classes d'âge. L'organisation culturelle reste marquée par la polygamie traduisant ainsi l'emprise de ce fait social dans cette communauté. Si l'exode rural des jeunes demeure une caractéristique des conséquences du JMC et des relations conflictuelles entre conjoints, il n'en demeure pas moins qu'il aura permis d'inverser la tendance dans les relations de genre favorisant ainsi l'accès de la femme à la terre et son autonomie dans le choix de la culture agricole produite et la pratique d'une activité génératrice de revenus. Cette avancée contraste avec le relâchement des relations intergénérationnelles aînés-cadets confinant les aînés dans un rôle passif et de second plan. Le JMC, en définitive, se présente comme un fait social dont le mérite serait certes d'avoir contribué à l'émancipation de la femme, mais avec pour conséquence d'avoir jeté les jalons d'un affaiblissement de la conscience collective.

\section{References:}

1. Amagou V., \& Brunin C. (1974). Le "plan cocotier" de Côte-d'Ivoire in Oléagineux, 29 ème année (7) : 359-364.

2. Amrizal I. (2003). Coconut statistical yearbook. Asian and Pacific Coconut Community. Jakarta (Indonesia). $192 \mathrm{p}$

3. Assa R. R. A., Konan J. L., Nemlin J., Prades A., Agbo N. \& Sie R.S. (2006). Diagnostic de la cocoteraie paysanne du littoral ivoirien. Sci. Nat. 3 (2) : 113-120.

4. Bene S., Courbet P., Dziwormu A., Salinier J., \& Mensah-Bonsu A. (2009). La filière du cocotier au Ghana et au Nigéria, OCL Vol. 16 $\mathrm{N}^{\circ} 2$ Mars-Avril 2009, pp 87-96. Article disponible sur le site http://www.ocl-journal.org ou http://dx.doi.org/10.1051/ocl.2009.0251 
5. Dollet M., Jannot C., Baudouin L., \& Ollivier J. C. (2009). Le cocotier en Afrique et la maladie du Jaunissement mortel, OCL Vol. 16 N² Mars-Avril, 74-75, Article disponible sur le site http://www.ocljournal.org ou http://dx.doi.org/10.1051/ocl.2009.0256

6. Gachanja G., Odhiambo Z., \& Musinga M. (2007). The coconut sub sector in Kenya. Baseline survey report. ABD-DANIDA/CDA. Mai 2007, 55 p.

7. Gaston J. (1910). Notes sur les Avikams de la lagune de Lahou et les Didas de la région du Bas-Bandama in : Bulletins et Mémoires de la Société d'anthropologie de Paris, VI Série. Tome 1, pp. 234-247.

8. INS [Institut National de la Statistique] (2014). Résultats globaux RGPH 2014, 10 p.

9. Legoupil J. C., \& Courbet P. (2008). Worldwide Coconut Production - Importance \& Impact of the Lethal Yellowing Disease: Justification and Objectives of the International Workshop, pp 19-26

10. Mariau D. (1999). Les maladies des cultures pérennes tropicales, Éditions Quae, coll. « Repères (CIRAD) », 287 p. (ISBN 9782876143401, ISSN 1251-7224)

11. N'da P. (2002). Méthodologie de la recherche, de la problématique à la discussion des résultats : comment réaliser un mémoire, une thèse en sciences sociales et en éducation ( $2^{\text {ème }}$ éd) Abidjan: Editions Universitaires de Côte d'Ivoire, 144 pages.

12. Nkaka N. (2013). «Grand-Lahou : Une mystérieuse maladie s’attaque aux cocotiers », http://www.linfodrome.com/economie/7380-grandlahou-une-mysterieuse-maladie-s-attaque-aux-cocotiers, consulté le 14 octobre 2016.

13. Ouvrier M., N. Zakra, A. Sangaré, \& Y. P. N'cho. (1995). Inventaire de la cocoteraie Ivoirienne. IDEFOR / DPO, Station Marc Delorme. Rapport de mission, $37 \mathrm{p}$.

14. Robert, A. D. \& Bouillarguet, A. (1997). L'analyse de contenu Paris : Presse Universitaire de France, Collection Le point des connaissances, 127 pages.

15. Ruf F., Bini S. \& Ampadu K. (2009). Stratégies des planteurs de cocotiers au Ghana face à la maladie du jaunissement mortel, OCL VOL. $16 \mathrm{~N}^{\circ} 2$ Mars-Avril 2009. Article disponible sur le site http://www.ocl-journal.org ou http://dx.doi.org/10.1051/ocl.2009.0252

16. umacher R. (1997). Méthodes qualitatives en recherche sociale sur les maladies tropicales: Rapport du matériel didactique Darda et N'Djamena, 6-24 octobre 1997, PNUD/ Banque Mondiale/OMS Programme spécial de recherche et de formation concernant les maladies tropicales (TDR), TDR/RCS/MQRS/02.1, 58 p. 
17. Yao A. A., Agneroh T. A., Pohe J., D’Almeida M. A. \& Zama P. (2012). Association d'organismes de type mycoplasmes avec le dépérissement mortel des cocotiers de Grand-Lahou en Côte d'Ivoire, International Journal of Biological Chemical and Sciences 6(3): 959984, Available online at http://ajol.info/index.php/ijbcs 